\title{
The Weight and Nonlinearity of 2-rotation Symmetric Cubic Boolean Function
}

\author{
Hongli Liu ${ }^{1}$ \\ ${ }^{1}$ Institute of Mathematics and Statistics, Zhejiang University of Finance and Economics, China \\ Correspondence: Hongli Liu, Institute of Mathematics and Statistics, Zhejiang University of Finance and Eco- \\ nomics, 18 XueYuan Street, Xiasha Higher Education Zone, Hangzhou 310018, China. Tel: 86-137-5827-3108. \\ E-mail: ooolhl@163.com
}

Received: March 25, 2015 Accepted: April 8, 2015 Online Published: May 23, 2015

doi:10.5539/jmr.v7n2p187 URL: http://dx.doi.org/10.5539/jmr.v7n2p187

The research is financed by the National Natural Science Foundation of China(Grant No:11302188), Zhejiang Provincial Natural Science Foundation of China (Grant No. LY14F010015).

\begin{abstract}
The conceptions of $\chi$-value and K-rotation symmetric Boolean functions are introduced by Cusick. K-rotation symmetric Boolean functions are a special rotation symmetric functions, which are invariant under the $k-t h$ power of $\rho$. In this paper, we discuss cubic 2-value 2-rotation symmetric Boolean function with $2 n$ variables, which denoted by $F^{2 n}\left(x^{2 n}\right)$. We give the recursive formula of weight of $F^{2 n}\left(x^{2 n}\right)$, and prove that the weight of $F^{2 n}\left(x^{2 n}\right)$ is the same as its nonlinearity.
\end{abstract}

Keywords: Rotation symmetric Boolean function, Nonlinearity, Weight, $\chi$-value

\section{Introduction}

Boolean functions have many applications in coding theory and cryptography. Rotation symmetric Boolean functions(RSBF) as invariant Boolean functions under rotation transform have been widely studied. Higher nonlinearity is a very important character of Boolean functions which are widely used in coding theory and S-box design. Rotation symmetric Boolean functions as a subclass of $K$ - rotation symmetric have not higher nonlinearity. So, $K$ - rotation symmetric Boolean functions which are the generalization of notion of rotation symmetric function were proposed by Selçk Kavut. The applications of the $k$-rotation symmetric $(k \geq 2)$ to coding theory and S-box design can be found in some papers. Cusick gave the definition of cubic 2 - rotation symmetric Boolean functions and used the notation $\left\{2-(1, r, s)_{2 n}: 2 n \geq s\right\}$ as the cubic monomial 2-rotation symmetric functions (denoted by 2 - functions). Cusick also described the affine equivalence of cubic MRS 2-rotation symmetric, and proved that the sequence of Hamming weights of $\left\{2-(1, r, s)_{2 n}: 2 n \geq s\right\}$ satisfies a linear recursion with integer coefficients. In this paper, we will give the recursion formula of Hamming weight of $\left\{2-(1,2,3)_{2 n}(2 n \geq 10)\right\}$ and prove that the nonlinearity of $\left\{2-(1,2,3)_{2 n}(2 n \geq 10)\right\}$ is the same as its weight.

\section{Preliminaries}

Let $\mathbb{F}_{2}=\{0,1\}$ be the binary field, $\mathbb{F}_{2}^{n}$ be the $n$-dimensional vector space of over $\mathbb{F}_{2}$. A Boolean function in $n$ variables can be defined as a map from $\mathbb{F}_{2}^{n}$ into $\mathbb{F}_{2}$, denoted by $f^{n}\left(x^{n}\right)$, or $f^{n}$ in brief, where $x^{n}=\left(x_{1}, x_{2}, \cdots, x_{n}\right)$. Every Boolean function $f^{n}$ has a unique polynomial representation (usually called the algebraic normal form (ANF)), and the degree of $f^{n}$ is the degree of this polynomial(deg $\left(f^{n}\right)$ in brief). If every term in the algebraic normal form of $f^{n}$ has the same degree, then the function is said to be homogeneous. A Boolean function $f^{n}$ is called affine, if $\operatorname{de} f\left(f^{n}\right)=1$. If $f^{n}$ is affine and homogeneous(i.e.the constant term is 0$), f^{n}$ is said to be linear. The truth table of $f^{n}$ is defined to be the binary sequence $v_{1}, v_{2}, \cdots, v_{2^{n}}$, where the bits $v_{1}=f((0,0, \cdots, 0)), v_{2}=$ $f((0,0, \cdots, 1)), \cdots, v_{2^{n}}=f((1,1, \cdots, 1))$. The Hamming weight of a Boolean function $f^{n}$ is defined as the number of nonzero coordinates in its truth table, denoted by $w t\left(f^{n}\right)$. The Hamming distance $d\left(f^{n}, g^{n}\right)$ between two Boolean functions $f^{n}$ and $g^{n}$ is defined as the number of their different coordinates, which equals the Hamming weight of their sum $f+g$, where + denotes the addition on $\mathbb{F}_{2}$. Two Boolean functions $f^{n}$ and $g^{n}$ in $n$ variables are said to be affine equivalent if there exists an invertible matrix $A$ with entries in $\mathbb{F}_{2}$ and $\mathbf{b} \in \mathbb{F}_{2}^{n}$ such that $f^{n}(\mathbf{x})=g^{n}(A \mathbf{x}+\mathbf{b})$. 
Definition 1 The nonlinearity $N L\left(f^{n}\right)$ of a Boolean function $f^{n}\left(x^{n}\right)$ is defined as

$$
N L\left(f^{n}\right)=\operatorname{Min}\left\{d\left(f^{n}\left(x^{n}\right), c^{n} \cdot x^{n}\right) \mid c^{n} \in \mathbb{F}_{2}^{n}\right\},
$$

where is the vector dot product.

It is easy to see that if $f^{n}$ and $g^{n}$ are affine equivalent, then $w t\left(f^{n}\right)=w t\left(g^{n}\right)$ and $N L\left(f^{n}\right)=N L\left(g^{n}\right)$. We say that the weight and nonlinearity are af fine invariants.

Definition 2 For a Boolean function $f^{n}\left(x^{n}\right)$. The Fourier transform of $f^{n}$ at $c^{n} \in \mathbb{F}_{2}^{n}$ is defined as

$$
\widehat{f^{n}}\left(c^{n}\right)=\sum_{x^{n} \in F_{2}^{n}}(-1)^{f^{n}\left(x^{n}\right)+c^{n} \cdot x^{n}} .
$$

Definition 3 A Boolean function $f^{n}\left(x^{n}\right)$ is called rotation symmetric if

$$
f^{n}\left(x_{1}, x_{2}, \cdots, x_{n}\right)=f^{n}\left(\rho\left(x_{1}, x_{2}, \cdots, x_{n}\right)\right), \text { for all }\left(x_{1}, x_{2}, \cdots, x_{n}\right) \in \mathbb{F}_{2}^{n},
$$

where $\rho\left(x_{1}, x_{2}, \cdots, x_{n-1}, x_{n}\right)=\left(x_{n}, x_{1}, x_{2}, \cdots, x_{n-1}\right)$.

If a monomial $x_{1} x_{2} x_{3}$ appears in a rotation symmetric Boolean function as a term then all monomials in the orbit of $x_{1} x_{2} x_{3}$ should appear in the function as terms. A rotation symmetric function is said to be monomial rotation symmetric $(M R S)$ if it is generated by applying powers of $\rho$ to a single monomial. We use the notation $(1, r, s)_{n}$ for the cubic MRS function in $n$ variables generated by the monomial $x_{1} x_{r} x_{s}$. A Boolean function is said to be $k$ - rotation symmetric if it is invariant under the $k-t$ th power of $\rho$ but not under any smaller power. A Boolean function is said to be monomial $k$ - rotation symmetric if it is generated by applying powers of $\rho^{k}$ to a single monomial. For brevity, we refer to these functions as $k-$ functions. In this paper, the cubic 2-functions shall be discussed. We use the notation $2-(1, r, s)_{2 n}$ for the cubic 2 -function in 2 n variables generated by the monomial $x_{1} x_{r} x_{s}$. If we assume $r<s \leq 2 n$ then the formula

$$
2-(1, r, s)_{2 n}=x_{1} x_{r} x_{s}+x_{3} x_{r+2} x_{s+2}+\cdots+x_{2 n-1} x_{r-2} x_{s-2} .
$$

is called a standard form of the above 2-function.

A monomial $[a, b, c]$ in a cubic 2 -function is said to be pure form, if $a, b, c$ are all even or odd. A monomial that is not pure form is said to be mixed form. It is obvious that every monomial of $2-(1, r, s)_{2 n}$ has the same form. A 2-function is said to be mixed form 2 - function if it's terms are mixed form. Otherwise, it is said to be pure form 2 - function.

Definition $4(\chi$-value $)$ Let $2-(1, r, s)_{2 n}$ be a mixed form 2 -function with monomial $[a, b, c](a<b<c)$. Assume $a$ is even (odd) and $b, c$ are odd(even). Then the $\chi-$ value for $2-(1, r, s)$ is defined as $\chi=c-b$.

Theorem 1 Two 2-functions $2-(1, r, s)_{2 n}$ and $2-(1, p, q)_{2 n}$ are affine equivalent by some permutation for all $n$ if and only if their $\chi$-values are equal.

Theorem 1 tells us that all 2 -values functions with $2 n$ variables have the same weights and nonlinearity. So, in the following section, we will discuss the weight and nonlinearity of 2 -values function $2-(1,2,3)_{2 n}$.

\section{The Weight of 2-values Function $F^{2 n}\left(x^{2 n}\right)$}

In this section, we shall study the recursive formula for weight of $2-(1,2,3)_{2 n}$. Firstly, we give the standard form of 2-values function $2-(1,2,3)_{2 n}$, denoted by $F^{2 n}\left(x^{2 n}\right)$ or $F^{2 n}$.

$$
F^{2 n}\left(x^{2 n}\right)=x_{1} x_{2} x_{3}+x_{3} x_{4} x_{5}+\cdots+x_{2 n-3} x_{2 n-2} x_{2 n-1}+x_{2 n-1} x_{2 n} x_{1} .
$$

If $T$ is a string, then $\bar{T}$ denotes the complemented string with 0 and 1 interchanged. If $X$ is a 4-bit block or a string of blocks, then $(X)_{s}$ or $X_{s}$ is the string obtained by concatenation of $s$ copies of $X$. The concatenation of two strings $u, v$ will be denoted by $u v$ or $u \| v$. Now we define two sets of 4-bit strings

$T_{1}=\{A=0,0,1,1 ; \bar{A}=1,1,0,0 ; B=0,1,0,1 ; \bar{B}=1,0,1,0 ; C=0,1,1,0 ; \bar{C}=1,0,0,1 ; D=0,0,0,0 ; \bar{D}=1,1,1,1\}$ and

$T_{2}=\{U=1,0,0,0 ; \bar{U}=0,1,1,1 ; V=0,0,0,1 ; \bar{V}=1,1,1,0 ; X=0,1,0,0 ; \bar{X}=1,0,1,1 ; Y=0,0,1,0 ; \bar{Y}=1,1,0,1\}$. 
We give the following result about the truth tables of monomials for $F^{2 n}\left(x^{2 n}\right)$.

Lemma 2 The truth table of any monomial for $F^{2 n}\left(x^{2 n}\right)$ is

$x_{i} x_{i+1} x_{i+2}=\left(D_{2^{2 n-i-2}}\left(D_{2^{2 n-i-3}}\left(D_{2^{2 n-i-4}} \bar{D}_{2^{n-i-4}}\right)\right)\right)_{2^{i-1}} \quad 1 \leq i \leq 2 n-5$, and $i$ is odd.

$x_{2 n-3} x_{2 n-2} x_{2 n-1}=(D D D A)_{2^{2 n-4}}$.

$x_{2 n-1} x_{2 n} x_{1}=D_{2^{2 n-3}} V_{2^{2 n-3}}$.

From Lemma 2, we give the following algorithm as the output of truth table for $F^{2 n}\left(x^{2 n}\right)$.

Algorithm 1

Step $5: h_{1}^{5} \leftarrow D D D A D D D A, h_{2}^{5} \leftarrow V V V Y V V V Y$.

Step $s: h_{i}^{s} \leftarrow\left(h_{i}^{s-2} \| \tilde{h}_{i}^{s-2}\right)_{2}, i=1,2$, for odd $s$.

Output $: H_{1} \leftarrow h_{1}^{2 n-1}, H_{2} \leftarrow \tilde{h}_{2}^{2 n-1}$, where $\tilde{h}_{i}^{s}$ is the string obtained from $h_{i}^{s}$ by complementing its last $2^{s-2}$ bits. Write $F^{2 n}=H_{1} \| H_{2}$.

From the above algorithm, we give the recursive relationship of weight for $F^{2 n}\left(x^{2 n}\right)$.

Theorem 3 The weight of Boolean function $F^{2 n}\left(x^{2 n}\right)$ satisfy

$$
w t\left(F^{2 n}\right)=2 w t\left(F^{2 n-2}\right)+4 w t\left(F^{2 n-4}\right)+2^{2 n-3} .
$$

Proof. Using Algorithm 1, we have

$$
w t\left(F^{2 n}\left(x^{2 n}\right)\right)=w t\left(H_{1}\right)+w t\left(H_{2}\right)=w t\left(h_{1}^{2 n-1}\right)+w t\left(\tilde{h}_{2}^{2 n-1}\right)
$$

and

$$
\begin{array}{ll}
h_{1}^{2 n-1}=h_{1}^{2 n-3} \tilde{h}_{1}^{2 n-3} h_{1}^{2 n-3} \tilde{h}_{1}^{2 n-3} & \tilde{h}_{1}^{2 n-1}=h_{1}^{2 n-3} \tilde{h}_{1}^{2 n-3} h_{1}^{2 n-3} \overline{\tilde{h}_{1}^{2 n-3}} \\
h_{1}^{2 n-3}=h_{1}^{2 n-5} \tilde{h}_{1}^{2 n-5} h_{1}^{2 n-5} \tilde{h}_{1}^{2 n-5} & \tilde{h}_{1}^{2 n-3}=h_{1}^{2 n-5} \tilde{h}_{1}^{2 n-5} h_{1}^{2 n-5} \overline{\tilde{h}_{1}^{2 n-5}} .
\end{array}
$$

Therefore,

$$
\begin{aligned}
w t\left(h_{1}^{2 n-1}\right) & =2\left(w t\left(h_{1}^{2 n-3}\right)+w t\left(\tilde{h}_{1}^{2 n-3}\right)\right) \\
& =2\left(4 w t\left(h_{1}^{2 n-5}\right)+2 w t\left(\tilde{h}_{1}^{2 n-5}\right)+2^{2 n-5}\right) \\
& =2\left(2 w t\left(h_{1}^{2 n-5}\right)+2\left(w t\left(h_{1}^{2 n-5}\right)+w t\left(\tilde{h}_{1}^{2 n-5}\right)\right)+2^{2 n-5}\right) \\
& =2\left(2 w t\left(h_{1}^{2 n-5}\right)+w t\left(h_{1}^{2 n-3}\right)+2^{2 n-5}\right) \\
& =4 w t\left(h_{1}^{2 n-5}\right)+2 w t\left(h_{1}^{2 n-3}\right)+2^{2 n-4} .
\end{aligned}
$$

Similarly, we have

$$
w t\left(\tilde{h}_{1}^{2 n-1}\right)=4 w t\left(\tilde{h}_{1}^{2 n-5}\right)+2 w t\left(\tilde{h}_{1}^{2 n-3}\right)+2^{2 n-4} .
$$

From (1), (2) and (3), we have

$$
\begin{aligned}
w t\left(F^{2 n}\left(x^{2 n}\right)\right) & =w t\left(h_{1}^{2 n-1}\right)+w t\left(\tilde{h}_{2}^{2 n-1}\right) \\
& =4 w t\left(h_{1}^{2 n-5}\right)+2 w t\left(h_{1}^{2 n-3}\right)+2^{2 n-4}+4 w t\left(\tilde{h}_{2}^{2 n-5}\right)+2 w t\left(\tilde{h}_{2}^{2 n-3}\right)+2^{2 n-4} \\
& =4 w t\left(F^{2 n-4}\right)+2 w t\left(F^{2 n-2}\right)+2^{2 n-3} .
\end{aligned}
$$

\section{The Nonliearity of $F^{2 n}\left(x^{2 n}\right)$}

Cusick and Stănică conjectured that the nonlinearity of cubic 1-values function $F^{n}\left(x^{n}\right)$ is the same as the weight, and Zhang et al. proved the conjecture. In this section, we shall prove the same result for $F^{2 n}\left(x^{2 n}\right)$, that is,

$$
w t\left(F^{2 n}\right)=N L\left(F^{2 n}\right)
$$


By the definitions of Fourier transform and Hamming weight, we can easily deduce that

$$
w t\left(F^{2 n}\left(x^{2 n}\right)\right)=\frac{1}{2}\left(2^{2 n}-\widehat{F^{2 n}(0)}\right) .
$$

Therefore, we can restate (4) as

$$
\widehat{F^{2 n}(0)}=\operatorname{Max}\left\{\mid \widehat{F^{2 n}\left(c^{2 n}\right)} \| c^{2 n} \in \mathbb{F}^{2 n}\right\} .
$$

On the other hand, the recursion formula of $\widehat{F^{2 n}(0)}$ can be obtained by applying the recursion formula of $w t\left(F^{2 n}\left(x^{2 n}\right)\right)$.

$$
\begin{aligned}
\widehat{F^{2 n}(0)} & =2^{2 n}-2 w t\left(F^{2 n}\right) \\
& =2^{2 n}-2 \cdot\left[2 w t\left(F^{2 n-2}\right)+4 w t\left(F^{2 n-4}\right)+2^{2 n-3}\right] \\
& =2\left[2^{2 n-1}-2 w t\left(F^{2 n-2}\right)-4 w t\left(F^{2 n-4}\right)-2^{2 n-3}\right] \\
& =2\left[2^{2 n-2}-2 w t\left(F^{2 n-2}\right)+2 \cdot 2^{2 n-4}-4 w t\left(F^{2 n-4}\right)\right] \\
& \left.=2\left[\widehat{F^{2 n-2}(0}\right)+2 \widehat{F^{2 n-4}(0)}\right] .
\end{aligned}
$$

Before giving the proof of (5), we need some notation:

$$
\begin{aligned}
& t_{2 n-1}=\sum_{1 \leq i \leq 2 n-3, i \text { is odd }} x_{i} x_{i+1} x_{i+2}, \\
& f_{1}^{2 n-1}\left(x_{1}, x_{2}, \cdots, x_{2 n-1}\right)=t_{2 n-1}, \\
& f_{2}^{2 n-1}\left(x_{1}, x_{2}, \cdots, x_{2 n-1}\right)=t_{2 n-1}+x_{1}, \\
& f_{3}^{2 n-1}\left(x_{1}, x_{2}, \cdots, x_{2 n-1}\right)=t_{2 n-1}+x_{2 n-1}, \\
& f_{4}^{2 n-1}\left(x_{1}, x_{2}, \cdots, x_{2 n-1}\right)=t_{2 n-1}+x_{2 n-1}+x_{1}, \\
& f_{5}^{2 n-1}\left(x_{1}, x_{2}, \cdots, x_{2 n-1}\right)=t_{2 n-1}+x_{2 n-1} x_{1} .
\end{aligned}
$$

Firstly, we give the following recursive relations about $\widehat{f_{i}^{2 n-1}}\left(c^{2 n-1}\right)$.

Lemma 4 For every $c^{2 n-1}=\left(c_{1}, c_{2}, \cdots, c_{2 n-1}\right) \in \mathbb{F}^{2 n-1}$, we have

$$
\begin{aligned}
& \widehat{f_{i}^{2 n-1}}\left(c^{2 n-1}\right)=\left(1+(-1)^{c_{2 n-1}}+(-1)^{c_{2 n-2}}\right) \widehat{f_{i}^{2 n-3}}\left(c^{2 n-3}\right)+(-1)^{c_{2 n-2}+c_{2 n-1}} \widehat{f_{i+2}^{2 n-3}}\left(c^{2 n-3}\right), i=1,2 . \\
& \widehat{f_{i}^{2 n-1}}\left(c^{2 n-1}\right)=\left(1-(-1)^{c_{2 n-1}}+(-1)^{c_{2 n-2}}\right) \widehat{f_{i}^{2 n-3}}\left(c^{2 n-3}\right)-(-1)^{c_{2 n-2}+c_{2 n-1}} \widehat{f_{i+2}^{2 n-3}}\left(c^{2 n-3}\right), i=3,4 . \\
& \widehat{f_{5}^{2 n-1}}\left(c^{2 n-1}\right)=\left(1+(-1)^{c_{2 n-2}}\right) \widehat{f_{1}^{2 n-3}}\left(c^{2 n-3}\right)+(-1)^{c_{2 n-1}} \widehat{f_{2}^{2 n-3}}\left(c^{2 n-3}\right)+(-1)^{c_{2 n-2}+c_{2 n-1}} \widehat{f_{4}^{2 n-3}}\left(c^{2 n-3}\right),
\end{aligned}
$$

where $c^{2 n-2}$ and $c^{2 n-3}$ are the first $2 n-2$ and $2 n-3$ bits of $c^{2 n-1}$.

proof We prove the relation for $i=1$, since the proof of the others are similar.

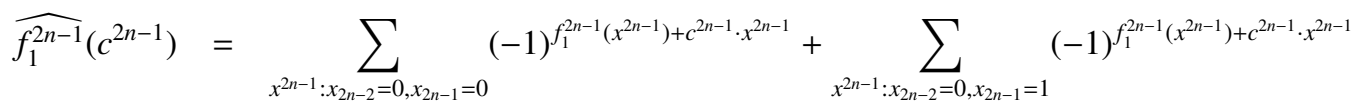

$$
\begin{aligned}
& +\sum_{x^{2 n-1}: x_{2 n-2}=1, x_{2 n-1}=0}(-1)^{f_{1}^{2 n-1}\left(x^{2 n-1}\right)+c^{2 n-1} \cdot x^{2 n-1}}+\sum_{x^{2 n-1}: x_{2 n-2}=1, x_{2 n-1}=1}(-1)^{f_{1}^{2 n-1}\left(x^{2 n-1}\right)+c^{2 n-1} \cdot x^{2 n-1}} \\
& =\sum_{x^{2 n-3}}(-1)^{f_{1}^{2 n-3}\left(x^{2 n-3}\right)+c^{2 n-3} \cdot x^{2 n-3}}+\sum_{x^{2 n-3}}(-1)^{f_{1}^{2 n-3}\left(x^{2 n-3}\right)+c^{2 n-3} \cdot x^{2 n-3}+c_{2 n-1}} \\
& +\sum_{x^{2 n-3}}(-1)^{f_{1}^{2 n-3}\left(x^{2 n-3}\right)+c^{2 n-3} \cdot x^{2 n-3}+c_{2 n-2}}+\sum_{x^{2 n-3}}(-1)^{f_{3}^{2 n-3}\left(x^{2 n-3}\right)+c^{2 n-3} \cdot x^{2 n-3}+c_{2 n-2}+c_{2 n-1}} \\
& =\widehat{f_{1}^{2 n-3}}\left(c^{2 n-3}\right)+(-1)^{c_{2 n-1}} \widehat{f_{1}^{2 n-3}}\left(c^{2 n-3}\right)+(-1)^{c_{2 n-2}} \widehat{f_{1}^{2 n-3}}\left(c^{2 n-3}\right)+(-1)^{c_{2 n-1}+c_{2 n-2}} \widehat{f_{3}^{2 n-3}}\left(c^{2 n-3}\right) \\
& =\left(1+(-1)^{c_{2 n-1}}+(-1)^{c_{2 n-2}}\right) \widehat{f_{1}^{2 n-3}}\left(c^{2 n-3}\right)+(-1)^{c_{2 n-1}+c_{2 n-2}} \widehat{f_{3}^{2 n-3}}\left(c^{2 n-3}\right) \text {. }
\end{aligned}
$$


From lemma 4, we can easily deduce the following corollary.

Corolary 5 For every $c^{2 n-1}=\left(c_{1}, c_{2}, \cdots, c_{2 n-1}\right) \in \mathbb{F}_{2}^{2 n-1}$, we have

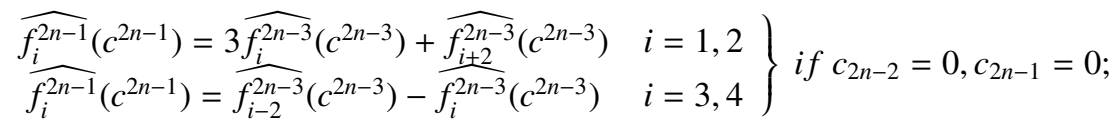

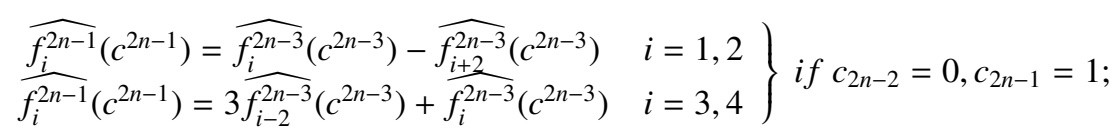

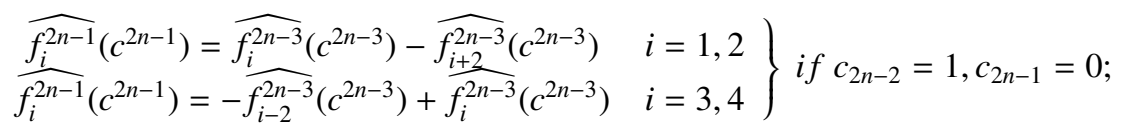

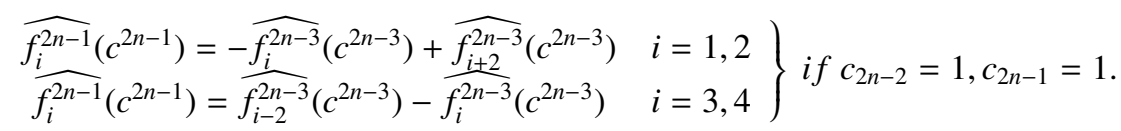

$$
\begin{aligned}
& \widehat{f_{5}^{2 n-1}}\left(c^{2 n-1}\right)=\left\{\begin{array}{cc}
2 \widehat{f_{1}^{2 n-3}}\left(c^{2 n-3}\right)+\widehat{f_{2}^{2 n-3}}\left(c^{2 n-3}\right)+\widehat{f_{4}^{2 n-3}}\left(c^{2 n-3}\right) & c_{2 n-2}=0, c_{2 n-1}=0 \\
2 \widehat{f_{1}^{2 n-3}}\left(c^{2 n-3}\right)-\widehat{f_{2}^{2 n-3}}\left(c^{2 n-3}\right)-\widehat{f_{4}^{2 n-3}}\left(c^{2 n-3}\right) & c_{2 n-2}=0, c_{2 n-1}=1 \\
\widehat{f_{2}^{2 n-3}}\left(c^{2 n-3}\right)-\widehat{f_{4}^{2 n-3}}\left(c^{2 n-3}\right) & c_{2 n-2}=1, c_{2 n-1}=0 \\
-\widehat{f_{2}^{2 n-3}}\left(c^{2 n-3}\right)+\widehat{f_{4}^{2 n-3}}\left(c^{2 n-3}\right) & c_{2 n-2}=1, c_{2 n-1}=1 .
\end{array}\right.
\end{aligned}
$$

Table 1. The values of $N L\left(F^{2 n}\right)$.

\begin{tabular}{cccccc}
\hline $2 n=8$ & $2 n=10$ & $2 n=12$ & $2 n=14$ & $2 n=16$ & $2 n=18$ \\
72 & 336 & 1472 & 6336 & 26752 & 111616 \\
\hline
\end{tabular}

Table 2. The values of $\widehat{F^{2 n}(0)}$.

\begin{tabular}{cccccc}
\hline $2 n=8$ & $2 n=10$ & $2 n=12$ & $2 n=14$ & $2 n=16$ & $2 n=18$ \\
112 & 352 & 1152 & 3712 & 12032 & 38912 \\
\hline
\end{tabular}

The following lemma give the properties of $\widehat{F^{2 n}}(0)$.

Lemma $6 \widehat{F^{2 n}}(0)$ satisfies the relationship: $\widehat{F^{2 n}}(0)>0$ and $\widehat{2 F^{2 n}}(0)<\widehat{F^{2 n+2}}(0)$.

proof We prove it by math induction. From Table 2, we can see the two results are true for $2 n=6,8,10,12,14,16,18$. Assume that, for an arbitrary $2 n$, the result is also true. Let's derive the correctness of conclusion for $2 n+2$ from this assumption.

From (6) and the assumption of induction, we have

$2 \widehat{F^{2 n+2}}(0)=2\left(2 \widehat{F^{2 n}}(0)+4 \widehat{F^{2 n-2}}(0)\right)<2 \widehat{F^{2 n+2}}(0)+4 \widehat{F^{2 n}}(0)=\widehat{F^{2 n+4}}(0)$

and

$\widehat{F^{2 n+2}}(0)=2 \widehat{F^{2 n}}(0)+4 \widehat{F^{2 n-2}}(0)>0$.

Which exactly means that the result holds for $2 n+2$.

Lemma 7 Let $c^{2 n-1}=\left(c_{1}, c_{2}, \cdots, c_{2 n-1}\right) \in \mathbb{F}_{2}^{2 n-1}$. If $c_{1}=1$, then

$$
\begin{gathered}
\left|\widehat{f_{i}^{2 n-1}}\left(c^{2 n-1}\right)\right|<\frac{1}{2} \widehat{F^{2 n}}(0),(i=1,5),\left|\widehat{f_{i}^{2 n-1}}\left(c^{2 n-1}\right)\right|<\frac{1}{4} \widehat{F^{2 n+2}}(0),(i=2,3,4) . \\
\left|\widehat{f_{1}^{2 n-1}}\left(c^{2 n-1}\right)\right|<\frac{1}{10} \widehat{F^{2 n+2}}(0),\left|\widehat{f_{2}^{2 n-1}}\left(c^{2 n-1}\right)\right|<\frac{3}{40} \widehat{F^{2 n+4}}(0) .
\end{gathered}
$$

proof 
When $c_{2 n-2}=0, c_{2 n-1}=0$, we have

$$
\begin{aligned}
& \widehat{f_{1}^{2 n-1}}\left(c^{2 n-1}\right)=3 \widehat{f_{1}^{2 n-3}}\left(c^{2 n-3}\right)+\widehat{f_{3}^{2 n-3}}\left(c^{2 n-3}\right) \\
& \widehat{f_{2}^{2 n-1}}\left(c^{2 n-1}\right)=3 \widehat{f_{2}^{2 n-3}}\left(c^{2 n-3}\right)+\widehat{f_{4}^{2 n-3}}\left(c^{2 n-3}\right) \\
& \widehat{f_{3}^{2 n-1}}\left(c^{2 n-1}\right)=\widehat{f_{1}^{2 n-3}}\left(c^{2 n-3}\right)-\widehat{f_{3}^{2 n-3}}\left(c^{2 n-3}\right) \\
& \widehat{f_{4}^{2 n-1}}\left(c^{2 n-1}\right)=\widehat{f_{2}^{2 n-3}}\left(c^{2 n-3}\right)+\widehat{f_{4}^{2 n-3}}\left(c^{2 n-3}\right) \\
& \widehat{f_{5}^{2 n-1}}\left(c^{2 n-1}\right)=2 \widehat{f_{1}^{2 n-3}}\left(c^{2 n-3}\right)+\widehat{f_{2}^{2 n-3}}\left(c^{2 n-3}\right)+\widehat{f_{4}^{2 n-3}}\left(c^{2 n-3}\right) .
\end{aligned}
$$

We prove it by math induction. The maximum values of $\left|\widehat{f_{i}^{11}}\left(c^{11}\right)\right|(i=1, \cdots, 5)$ can be obtained with the help of Matlab soft, which are 352, 672, 672, 672, 352. From Talbe 2, we can see $\widehat{f_{i}^{11}}\left(c^{11}\right)(i=1,5)<\frac{1}{2} \widehat{F^{12}}(0), \widehat{f_{i}^{11}}\left(c^{11}\right)(i=$ $2,3,4)<\frac{1}{4} \widehat{F^{14}}(0), \widehat{f_{1}^{11}}\left(c^{11}\right)<\frac{1}{10} \widehat{F^{14}}(0)$, and $\widehat{f_{2}^{11}}\left(c^{11}\right)<\frac{3}{40} \widehat{F^{16}}(0)$.

Suppose the results are true for $2 n-1(n \geq 6)$, we prove that it is true for $2 n+1$.

$$
\begin{aligned}
& \left|\widehat{f_{1}^{2 n+1}}\left(c^{2 n+1}\right)\right|=\left|3 \widehat{f_{1}^{2 n-1}}\left(c^{2 n-1}\right)+\widehat{f_{3}^{2 n-1}}\left(c^{2 n-1}\right)\right| \\
& =\left|\widehat{f_{1}^{2 n-1}}\left(c^{2 n-1}\right)+\widehat{f_{1}^{2 n-1}}\left(c^{2 n-1}\right)+\widehat{f_{3}^{2 n-1}}\left(c^{2 n-1}\right)\right| \\
& =\left|2 \widehat{f_{1}^{2 n-1}}\left(c^{2 n-1}\right)+4 \widehat{f_{1}^{2 n-3}}\left(c^{2 n-3}\right)\right| \\
& \leq 2\left|\widehat{f_{1}^{2 n-1}}\left(c^{2 n-1}\right)\right|+4\left|\widehat{f_{1}^{2 n-3}}\left(c^{2 n-3}\right)\right| \\
& <\frac{1}{2}\left(2 \widehat{F^{2 n}}(0)+4 \widehat{F^{2 n-2}}(0)\right)\left(<\frac{1}{10}\left(2 \widehat{F^{2 n+2}}(0)+4 \widehat{F^{2 n}}(0)\right)\right) \\
& =\frac{1}{2} \widehat{F^{2 n+2}}(0)\left(=\frac{1}{10} \widehat{F^{2 n+4}}(0)\right) \text {. } \\
& \left|\widehat{f_{2}^{2 n+1}}\left(c^{2 n+1}\right)\right|=\left|3 \widehat{f_{2}^{2 n-1}}\left(c^{2 n-1}\right)+\widehat{f_{4}^{2 n-1}}\left(c^{2 n-1}\right)\right| \\
& =\left|\widehat{f_{2}^{2 n-1}}\left(c^{2 n-1}\right)+\widehat{f_{2}^{2 n-1}}\left(c^{2 n-1}\right)+\widehat{f_{4}^{2 n-1}}\left(c^{2 n-1}\right)\right| \\
& =\left|2 \widehat{f_{2}^{2 n-1}}\left(c^{2 n-1}\right)+4 \widehat{f_{2}^{2 n-3}}\left(c^{2 n-3}\right)\right| \\
& \leq 2\left|\widehat{f_{2}^{2 n-1}}\left(c^{2 n-1}\right)\right|+4\left|\widehat{f_{2}^{2 n-3}}\left(c^{2 n-3}\right)\right| \\
& <\frac{1}{4}\left(2 \widehat{F^{2 n+2}}(0)+4 \widehat{F^{2 n}}(0)\right)\left(<\frac{3}{40}\left(2 \widehat{F^{2 n+4}}(0)+4 \widehat{F^{2 n+2}}(0)\right)\right) \\
& =\frac{1}{4} \widehat{F^{2 n+4}}(0)\left(\left(=\frac{3}{40} \widehat{F^{2 n+6}}(0)\right)\right. \text {. } \\
& \left|\widehat{f_{3}^{2 n+1}}\left(c^{2 n+1}\right)\right|=\left|\widehat{f_{1}^{2 n-1}}\left(c^{2 n-1}\right)-\widehat{f_{3}^{2 n-1}}\left(c^{2 n-1}\right)\right| \\
& \left.=\mid 2 \widehat{f_{1}^{2 n-1}}\left(c^{2 n-1}\right)-\widehat{\left(\widehat{f_{1}^{2 n-1}}\right.}\left(c^{2 n-1}\right)+\widehat{f_{3}^{2 n-1}}\left(c^{2 n-1}\right)\right) \mid \\
& =\left|2 \widehat{f_{1}^{2 n-1}}\left(c^{2 n-1}\right)-4 \widehat{f_{1}^{2 n-3}}\left(c^{2 n-3}\right)\right| \\
& \leq 2\left|\widehat{f_{1}^{2 n-1}}\left(c^{2 n-1}\right)\right|+4\left|\widehat{f_{1}^{2 n-3}}\left(c^{2 n-3}\right)\right| \\
& <\frac{1}{2}\left(2 \widehat{F^{2 n}}(0)+4 \widehat{F^{2 n-2}}(0)\right) \\
& =\frac{1}{2} \widehat{F^{2 n+2}}(0) \leq \frac{1}{4} \widehat{F^{2 n+4}}(0) \text {. } \\
& \left|\widehat{f_{4}^{2 n+1}}\left(c^{2 n+1}\right)\right|=\left|\widehat{f_{2}^{2 n-1}}\left(c^{2 n-1}\right)-\widehat{f_{4}^{2 n-1}}\left(c^{2 n-1}\right)\right| \\
& \left.=\mid 2 \widehat{f_{2}^{2 n-1}}\left(c^{2 n-1}\right)-\widehat{\left(f_{2}^{2 n-1}\right.}\left(c^{2 n-1}\right)+\widehat{f_{4}^{2 n-1}}\left(c^{2 n-1}\right)\right) \mid
\end{aligned}
$$




$$
\begin{aligned}
& =\left|2 \widehat{f_{2}^{2 n-1}}\left(c^{2 n-1}\right)-4 \widehat{f_{2}^{2 n-3}}\left(c^{2 n-3}\right)\right| \\
& \leq 2\left|\widehat{f_{2}^{2 n-1}}\left(c^{2 n-1}\right)\right|+4\left|\widehat{f_{2}^{2 n-3}}\left(c^{2 n-3}\right)\right| \\
& <\frac{1}{4}\left(2 \widehat{F^{2 n+2}}(0)+4 \widehat{F^{2 n}}(0)\right) \\
& =\frac{1}{4} \widehat{F^{2 n+4}}(0) . \\
& \left|\widehat{f_{5}^{2 n+1}}\left(c^{2 n+1}\right)\right|=\left|2 \widehat{f_{1}^{2 n-1}}\left(c^{2 n-1}\right)+\widehat{f_{2}^{2 n-1}}\left(c^{2 n-1}\right)+\widehat{f_{4}^{2 n-1}}\left(c^{2 n-1}\right)\right| \\
& =\left|2 \widehat{f_{1}^{2 n-1}}\left(c^{2 n-1}\right)+4 \widehat{f_{2}^{2 n-3}}\left(c^{2 n-3}\right)\right| \\
& \leq 2\left|\widehat{f_{1}^{2 n-1}}\left(c^{2 n-1}\right)\right|+4\left|\widehat{f_{2}^{2 n-3}}\left(c^{2 n-3}\right)\right| \\
& <\frac{1}{5} \widehat{F^{2 n+2}}(0)+\frac{3}{10} \widehat{F^{2 n+2}}(0) \\
& =\frac{1}{2} \widehat{F^{2 n+2}}(0) .
\end{aligned}
$$

When $c_{2 n-2}=1, c_{2 n-1}=0$, we have

$$
\begin{aligned}
& \left|\widehat{f_{5}^{2 n+1}}\left(c^{2 n+1}\right)\right|=\left|\widehat{f_{2}^{2 n-1}}\left(c^{2 n-1}\right)-\widehat{f_{4}^{2 n-1}}\left(c^{2 n-1}\right)\right| \\
& \leq\left|\widehat{f_{2}^{2 n-1}}\left(c^{2 n-1}\right)\right|+\left|\widehat{f_{4}^{2 n-1}}\left(c^{2 n-1}\right)\right| \\
& <\frac{1}{4} \widehat{F^{2 n+2}}(0)+\frac{1}{4} \widehat{F^{2 n+2}}(0) \\
& =\frac{1}{2} \widehat{F^{2 n+2}}(0) .
\end{aligned}
$$

The others cases are similar.

From definition $3, \widehat{F^{2 n}}\left(c_{1}, c_{2}, \cdots, c_{2 n}\right)=\widehat{F^{2 n}}\left(c_{2 n}, c_{1}, \cdots, c_{2 n-1}\right)$, for any $c^{2 n}=\left(c_{1}, c_{2}, \cdots, c_{2 n}\right) \in \mathbb{F}_{2}^{2 n}$. Without lost of generality, if $c^{2 n} \neq 0$, we can assume that $c_{1} \neq 0$. Using lemma 4 , we have the following theorem.

Theorem 8 For all $c^{2 n}=\left(c_{1}, c_{2}, \cdots, c_{2 n}\right) \neq 0$ and $n \geq 6$, we have

$$
\left|\widehat{F^{2 n}}\left(c^{2 n}\right)\right|<\widehat{F^{2 n}}(0) \text {. }
$$

proof We factor $\widehat{F^{2 n}}\left(c^{2 n}\right)$ into two sub-functions.

$\left|\widehat{F^{2 n}}\left(c^{2 n}\right)\right|=\left|\widehat{f_{1}^{2 n-1}}\left(c^{2 n-1}\right)+\widehat{f_{5}^{2 n-1}}\left(c^{2 n-1}\right)\right| \leq\left|\widehat{f_{1}^{2 n-1}}\left(c^{2 n-1}\right)\right|+\left|\widehat{f_{5}^{2 n-1}}\left(c^{2 n-1}\right)\right|<\frac{1}{2} \widehat{F^{2 n}}(0)+\frac{1}{2} \widehat{F^{2 n}}(0)=\widehat{F^{2 n}}(0)$.

Theorem 8 tells us that the nonlinearity of $F^{2 n}\left(x^{2 n}\right)$ is the same as its weight.

\section{Conclusion}

This paper gives the recursive formula of weight about 2-values cubic Boolean functions with $2 n$ variables, and proves that the weight of $F^{2 n}$ is the same as its nonlinearity. The recursive formula of weight about 2tvalues $(\mathrm{t}=2,4, \cdots)$ cubic Boolean functions can be discussed and the relationship of weight between 2-value and $2 \mathrm{t}$-value functions can also be studied.

\section{Acknowledgements}

The work was supported by the National Natural Science Foundation of China(Grant No:11302188), Zhejiang Provincial Natural Science Foundation of China (Grant No. LY14F010015).

\section{References}

Cusick, T. W., \& Johns, B. (2014). Theory of 2-rotation symmetric cubic Boolean functions: Des. Codes Cryptogr. http://dx.doi.org/10.1007/s10623-014-9964-2

Cusick, T. W., \& Padgett, D. (2012). A recursive formula for weights of Boolean rotation symmetric functions: Discrete Applied Mathematics 160, 391-397. http://dx.doi.org/10.1016/j.dam.2011.11.006 
Cusick, T. W., \& Stanica, P. (2009). Cryptographic Boolean functions:Academic Press. San Didgo.

Kavut, S. (2012). Results on rotation-symmetric S-boxes. Information Sciences, 201, 93-113. http://dx.doi.org/10.1016/j.ins.2012.02.030

Kavut, S., \& Ycel, M. D. (2010). 9-variable Boolean functions with nonlinearity 242 in the generalized rotation symmetric class. Information Sciences, 208(4), 341-350. http://dx.doi.org/10.1016/j.ic.2009.12.002

Kim, H., Park, S. M., \& Hahn, S. G. (2009). On the weight and nonlinearity of homogeneous rotation symmetric Boolean functions of degree 2. Discrete Applied Mathematics, 157, 428-432. http://dx.doi.org/10.1016/j.dam.2008.06.022

\section{Copyrights}

Copyright for this article is retained by the author(s), with first publication rights granted to the journal.

This is an open-access article distributed under the terms and conditions of the Creative Commons Attribution license (http://creativecommons.org/licenses/by/3.0/). 